\title{
BMJ Open Should GPs have direct access to imaging for headache? A qualitative study of patients' views in the UK
}

\author{
Alison McKinlay, ${ }^{\oplus 1}$ Raphael Underwood, ${ }^{\oplus 1}$ Gabriella Wojewodka, ${ }^{1}$ Asif Mazumder, ${ }^{2}$ \\ Rachael Kilner, ${ }^{3}$ Leone Ridsdale ${ }^{1}$
}

To cite: McKinlay A, Underwood R, Wojewodka $G$ et al. Should GPs have direct access to imaging for headache? A qualitative study of patients' views in the UK. BMJ Open 2019;9:e029376. doi:10.1136/ bmjopen-2019-029376

- Prepublication history and additional material for this paper are available online. To view these files, please visit the journal online (http://dx.doi. org/10.1136/bmjopen-2019029376).

Received 23 January 2019 Revised 19 June 2019 Accepted 1 July 2019

Check for updates

(C) Author(s) (or their employer(s)) 2019. Re-use permitted under CC BY-NC. No commercial re-use. See rights and permissions. Published by BMJ.

${ }^{1}$ Department of Basic and Clinical Neuroscience, Institute of Psychiatry, Psychology and Neuroscience, King's College London, London, UK

${ }^{2}$ Neuroradiology Group, Department of Radiology, Lambeth Wing, Guy's and St Thomas' Hospitals NHS Foundation Trust, London, UK ${ }^{3}$ Department of Neurology, King's College Hospital, London, UK

Correspondence to Professor Leone Ridsdale; leone.ridsdale@kcl.ac.uk

\section{ABSTRACT}

Objective We aimed to describe patients' views of a new referral pathway of general practitioner (GP) direct access to MRI, versus imaging after referral to a specialist. Design This qualitative study involved 20 semistructured interviews. Twenty patients (10 from each pathway) were purposively recruited and interviewed to describe their attitudes.

Setting A neurology headache clinic and neuroradiology services from the boroughs of Southwark and Lambeth in South London, UK.

Participants Twenty patients were involved in this study. Results Over half of the participants felt relieved once they received their scan results, while some remained uncertain about the underlying cause of their symptoms. Some participants described a long wait to see a specialist. Others described a long wait time to receive scan results, especially from their GP. Spontaneous reduction in headache symptoms occurred for some participants and for others, normal imaging results allowed them to focus more on symptom management.

Conclusion Relief was reported especially when scan results had been explained clearly and without too much delay. Those with continuing pain focused on how to get relief from symptoms. Patient experience might be improved with clearer information from GPs about how patients can access results, standard reporting procedures and closer liaison between neuroradiology and GPS.

\section{INTRODUCTION}

In the UK, 3\% of adults visit their general practitioner (GP) for headache each year. ${ }^{1}$ Patients who consult their GP for headache require a diagnosis, with advice on pain and lifestyle management. ${ }^{2}$ Aside from neurologists, doctors report lack of confidence in diagnosing and managing neurological conditions in primary care (PC). Nevertheless, GPs manage $97 \%$ of headache consulters, without referral to a specialist. ${ }^{1}$

Of those who consult GPs for headache, $2 \%$ are then referred to neurology clinics ${ }^{1}$ typically when symptoms persist and red flags are indicated. The high prevalence of headache results in it being the most common reason for new referral to neurology clinics, accounting

\section{Strengths and limitations of this study}

This is the first study to describe patient experiences of general practitioner (GP) direct access to imaging for headache, compared with imaging requested by a neurologist.

- Our results highlight that GP direct access to scans was quicker than specialist referral for scanning; however, potential benefits of GP access were reduced by unclear and slow communication of results.

- The qualitative study included views of 20 patients which is lower than a survey would provide, but the quality of our data is richer in detail.

for a quarter to a third of new appointments. ${ }^{4}$ However, those referred do not have more severe headaches. ${ }^{5}$ Referred patients tend to report more anxiety about their symptoms, have consulted their GP repeatedly, and want imaging to rule out serious pathology. ${ }^{56}$ With long waiting times to see specialists, the question can demand for diagnostic services be managed more directly arises.

GPs are moving from symptom gatekeepers to facilitators of early diagnosis and management for an increasing number of serious conditions. Evidence suggests that GPs can use direct-access imaging, and potentially reduce unnecessary specialist referral. ${ }^{7-9}$

An argument against GP direct access to imaging is that imaging is not a panacea and it does not necessarily resolve diagnostic uncertainty. When there are non-specific findings, as in $2 \%$ of MRI cases, scans can actually lead to more anxiety and uncertainty. ${ }^{1011}$ Unnecessary imaging may cause diagnostic delays and increase anxiety. ${ }^{2}$ MRI does not necessarily show all causes of headache and serious pathology may remain unidentified even with a scan. On the other hand, after a normal scan undertaken in specialist care, patients, especially those who have few anxiety/depression symptoms prior to imaging, do report relief. ${ }^{12}$ 


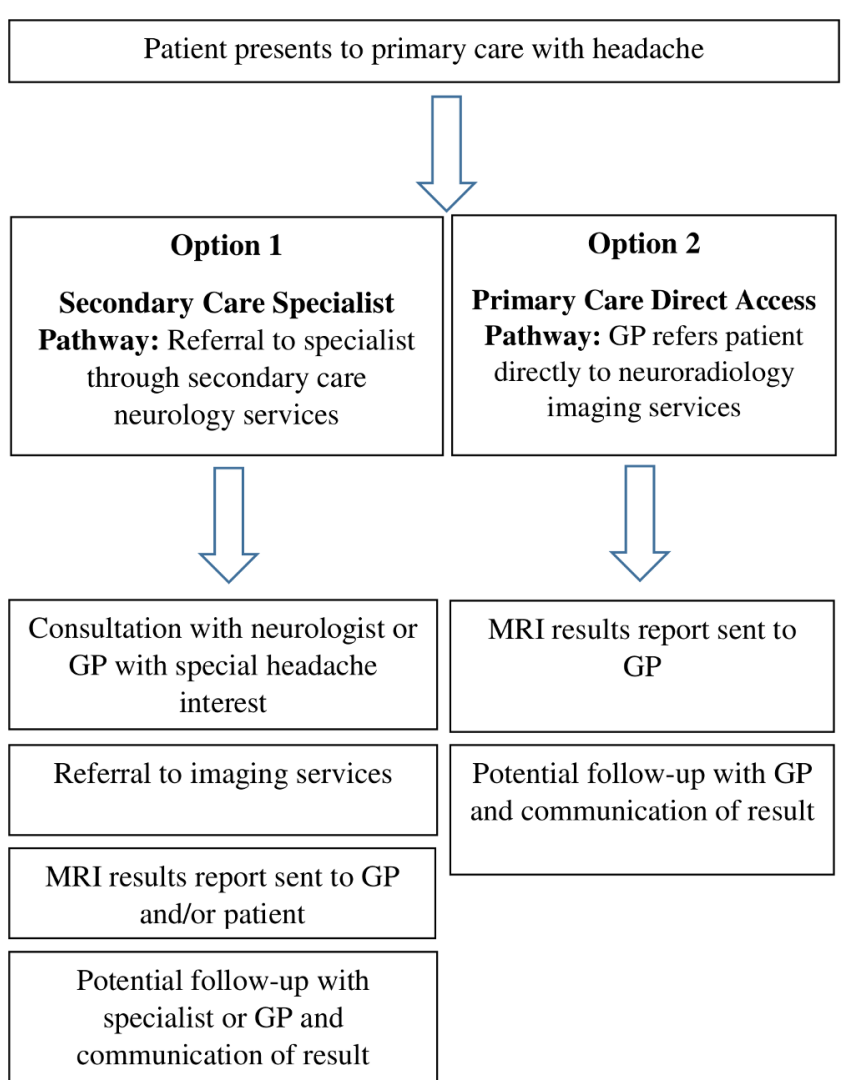

Some possible outcomes: Patient satisfaction/dissatisfaction, (non)-relief of uncertainty about underlying causes, symptom remission/persistence

Figure 1 Usual and alternative referral pathway for headache patients. GP, general practitioner; PC, primary care; SC, secondary care.

In a project designed to increase access to investigations, we are evaluating the option of GP direct access to MRI for headache in South London, UK (figure 1). In the first part of the study, we interviewed 20 GPs to elicit their views about this and the ability to use the direct-access service for headache. ${ }^{13}$ A training session delivered by a GP with specialist training in headache was available to GPs as part of the study. Those attending the session said it improved their knowledge, skills and confidence to implement the direct-access service into their practice. ${ }^{13}$ Most GPs reported that in the past a normal scan resolved uncertainty, and helped them progress to discussion of medication and psychosocial issues with patients.

The current study follows on from GP views, ${ }^{13}$ where we now aim to describe the patient experience of direct access to MRI (PC, pathway), compared with the traditional route through a specialist (secondary care (SC), pathway; figure 1). We were particularly interested in opinions regarding appointment length, value of early relief from direct-access imaging and preference for the specialist or GP.

\section{METHODS}

\section{Study design}

We conducted an observational, opt-in study, using qualitative methodology with a deductive analytic approach. ${ }^{14}$ Semistructured, one-off, qualitative interviews were conducted with 20 patients, purposively recruited, with 10 participants from each referral pathway. Based on our past research ${ }^{13}$ and qualitative methodology literature,${ }^{15}$ we estimated data saturation to occur after 10 participant interviews per referral pathway.

Participation was voluntary with no incentives offered. Data collected were self-report. Researchers did not access any medical records about participants. Researchers verbally discussed the study, provided written information and obtained verbal and written informed consent from participants at the start of each interview. For the write-up of this research, we implemented the Consolidated criteria for Reporting Qualitative research (COREQ) studies. ${ }^{16}$

\section{Patient and public involvement}

The study design was conceptualised by RU, LR, AM and RK, with input from a migraine user group charity. Patients' expectations when requesting specialist referral for headaches and their views of care have been described previously by members in our research group. ${ }^{17}$ There was no subsequent patient involvement in the design of this study.

\section{Participants and recruitment}

Participants were recruited through King's Health Partners from the Boroughs of Southwark and Lambeth in South London, UK. For the SC pathway, participants were recruited through a general neurology clinic where a specialist gave eligible patients information about the study. For the PC pathway, recruitment occurred through neuroradiology services, where interested patients could request for a researcher to contact them. Further details on recruitment methods are available in online supplementary material 1 .

Inclusion criteria were $\geq 18$ years old, treated in PC, received a scan either by SC or PC referral and either neurologist diagnosed headache or self-reported headache for $\geq 3-6$ months. Exclusion criteria were not fluent in English, severe psychiatric illness, new or sudden onset headache $\leq 3$ months or significant abnormality reported on MRI. We made one protocol amendment to interview all eligible patients at least 2 months after having a scan (refer to online supplementary material). Hospital staff identified potentially eligible patients for the study. This was reconfirmed by the researcher by checking their eligibility criteria when obtaining written informed consent.

Fifty-two patients registered an interest in the study. Researchers were unable to subsequently contact 24 patients. As per study protocol, two were excluded due 
to non-normal scans, six declined the interview invitation or were unable to attend.

An interview schedule was developed based on previous research. ${ }^{617}$ Interview questions were arranged as a list of predetermined themes (eg, managing uncertainty, expertise; refer to online supplementary material $2)$. The SC pathway interviews were held first (P1-P10), PC direct access second (P11-P20).

\section{Interviews}

One male (RU) and one female (CP) researcher conducted interviews. RU has a PhD in psychology and CP has an MSc in psychology. Both have prior experience of qualitative interviews. No prior relationships existed between any participant and the researchers.

Interviews were held between June 2016 and August 2017, ranging from 28 to $117 \mathrm{~min}$ in duration. One-on-one interviews were conducted in a location convenient to the participant, such as their home or a public place. Interviews were audio recorded and later transcribed using a third-party transcription service. Standard data protection methods were followed. No field notes were taken during interviews. Transcripts were anonymised and consent forms stored in a locked cabinet. Contact information provided was used only to organise interviews and stored in a password-protected file.

\section{Analysis}

The data were analysed thematically ${ }^{14}$ and managed using QSR NVivo V.11 (QSR International, London, UK). Two researchers (AMc, RU) read all transcripts, which were coded based on emergent patterns that were then organised by theme. Code lists and schematics were discussed and agreed with all coauthors. Themes more commonly raised by participants are reported here.

\section{RESULTS}

\section{Participant characteristics}

Ten participants in the group had been referred to SC services and 10 patients had been offered PC direct-access imaging through their GP. All participants attended one interview (F: 11, M: 9; age range: 20-72, median: 41). Seven participants (SC: 3, PC: 4) had also visited emergency departments for headache and one participant had been admitted to hospital for suspected thunderclap headaches. Three participants (SC: 2, PC: 1) indicated they had incidental findings on their scan.

\section{Theme 1: the effect of time on patient satisfaction}

Long wait for specialist appointment

Wait times were about 12 weeks, ranging from 2 weeks to 5 months. Of the 10 who were referred to SC, 7 participants said they waited a long time for their specialist appointment.
I'd been referred on the twoweeks' pathway* [in June], and then the two week pathway for the MRI scan... and then the letter I got was, [it] asked me to come back for a follow-up appointment, but in December. (P10)

$$
\text { *pathway for suspected cancer }
$$

Long wait times for scan results

Eight participants (SC: 2, PC: 6) said they had a long wait for their scan results. This was more apparent in the PC than SC pathway. Of this group, four participants in the PC pathway did not have their results 2 months after their scan. Several participants described a mismatch between what they were told or expected from their referral, and what actually occurred.

They told me it takes three weeks, but I was expecting like, just a couple of weeks, not a couple of months. (P16)

\section{Consultation length sufficient}

In the UK, specialists in SC commonly have twice as much time available to see patients with new problems compared with GPs. Nonetheless an equal number of participants in each pathway (SC: 4, PC: 4) said their consultation length was satisfactory. Many described an awareness that their GP had time constraints, and therefore tried to optimise their use of time in the consultation.

It's not necessarily about the length of time but it's about the GP creating an atmosphere and an environment where you feel like you can talk freely and not that you're under pressure. (P11)

\section{Relief of uncertainty}

Eleven participants (SC: 8, PC: 3) said they felt reassurance after receiving their scan results, mostly from the SC pathway. For many, normal results allayed fears about serious pathology. One of these participants said having a name for the condition helped to minimise fear and uncertainty $(\mathrm{P} 7)$.

\section{Theme 2: communication of results}

\section{Clear explanation}

More participants in the SC group said they received a clear explanation from medical staff about their scan results (SC: 7, PC: 3). However, this difference is likely to have been affected by the fact that four participants from the PC pathway had not received their scan results 2 months after their scan. Many said it helped to have further details about the potential cause of their symptoms.

Once the report was written, it was clear that it was more something to do with the eyes than to do with something within the head... So, I thought that 
explanation matched my experience, and I was happy to leave it at that. (P13)

\section{Communication difficulty}

Six participants (SC: 3, PC: 3) said they experienced difficulty with doctor-patient communication. For four participants, neuroimaging results were difficult to understand or unhelpful. Three of these participants said their results report contained medical jargon.

I didn't understand any of it, but I just remembered that now the pituitary faucet is relatively empty and I was like, 'What does that mean?... What's the pituitary? - I didn't know. (P5)

\section{Theme 3: continued uncertainty}

Five participants (SC: 3, PC: 2) said ruling out pathology was a relief, but when a cause could not be determined, persistent headache symptoms were associated with continued uncertainty. For one participant, not having an explanation of the underlying cause was framed as a lack of validation of their experience:

I feel like I'm left with, well, maybe there's something there that nobody seems to think is there, except me. (P11)

\section{Theme 4: symptom management}

\section{Spontaneous symptom reduction after scan}

Four participants (SC: 1, PC: 3), mostly in the PC pathway, experienced symptom reduction after their scan without additional intervention.

The uncertainty wasn't really terrible because I knew that if it was something terrible, it would be getting worse, and it wasn't. It was getting better. (P10)

\section{Importance of pain management}

After receiving an MRI scan, eight participants (SC: 4, PC: 4) said they were still worried about ongoing pain. Two said pain reduction was now the most important aspect of their care.

I think the brain scan to me wasn't even that important. To me, the important thing to me was to get rid of the pain. (P6)

\section{Theme 5: specialist or GP preference Specialist expertise}

Fifteen participants said they had appreciated (SC: 9) or would have liked (PC: 6) input from a specialist. The specialist was seen to have headache-specific knowledge that they felt their GP did not. For example, a participant seen in PC only said the following:

The scan would be avoided if you get seen by a specialist, because they have got more experience and they might have realised my issue wasn't with anything in my head, it was more a muscle problem, and a stress problem than anything else. (P20)

\section{GP management}

Seven participants (SC: 2, PC: 5) indicated that despite the perceived value of specialist knowledge, they would nevertheless prefer seeing their GP for headache-related care. Four of these participants said their GP was a consistent point of contact, with whom rapport and trust had been previously established. One participant felt their scan referral would be processed more quickly through the GP (P9), and another reported transportation difficulty with accessing specialist services (P10).

... it's better if you see one doctor, he knows you and he knows your problem. (P18)

\section{DISCUSSION}

\section{Summary}

We aimed to describe similarities and differences between participants' experiences of two referral pathways. Overall, we found that there were more similarities than differences. The same number of patients described feeling satisfied with their headache consultation times and more than half of the sample said they felt relief of uncertainty after receiving their results. However, despite reducing anxiety regarding serious pathology, almost half still experienced pain. The data suggested that waiting-list delays in seeing a specialist could prolong patients' anxiety and uncertainty. A potential benefit of GP direct access to imaging might be avoidance of this delay. However, the potential benefits were to some extent vitiated by perceived delays in receiving results, particularly from GPs. Patient satisfaction and relief were reported when scan results were explained clearly and quickly. This was more commonly reported after seeing a specialist, where results were typically given during the follow-up appointment.

Non-specific findings and communication difficulty were a problem for a minority in both pathways. After imaging nearly half were more focused on pain management. Specialist expertise was valued by more participants, but many valued management in PC due to a pre-established, ongoing relationship with the GP, and ease of access compared with neurology services.

\section{Strengths and limitations}

The main strength of this study is that, to our knowledge, no published research has described patient experiences of direct access to imaging for headache. A weakness of the study relates to recruitment in a limited time period. The SC pathway recruitment was completed first, as there was delay in the new PC-access imager coming into operation. SC pathway participants were interviewed once they had received their report, with no maximum time limit. In interviewing the second group of PC direct-access participants, we had research worker time constraints, 
and included four participants who had not received their scan results. PC pathway participants were interviewed at maximum 2 months after their scan, a sufficient time for reports to reach GPs.

\section{Implications for research and practice}

Prior studies have suggested potential advantages and disadvantages to open-access imaging in PC for GPs. Advantages include speed of diagnosis or, more commonly, exclusion of serious pathology, diagnostic yield from imaging which could help with subsequent management and avoidance of unnecessary referrals to SC. ${ }^{7918}{ }^{19}$ A disadvantage is the management of incidental findings; however, this can occur regardless of whether patients are referred for imaging by specialists or GPs. ${ }^{812}$ We found previously that GPs do report difficulty in managing these,$^{13}$ which suggests this to be an area of medical education that could be targeted in future trainee cohorts.

\section{Managing resources}

Given that resources and capacity are limited, a fear among policy-makers and providers is that demand for imaging and referral will rise from patients. GPs raised the lack of specialist input as another disadvantage of direct access, which may hinder in reassuring patients after a normal scan. ${ }^{13}$ In our previous study, we found that added training with an educational course regarding headache and direct imaging increased GP confidence in managing headache. ${ }^{13}$ This option may help GPs feel equipped to manage the discussion of normal scan results (or avoid a scan altogether). Our results show that some patients would like specialist input, but others preferred to manage their care with a GP.

\section{GP training}

Further clinical training may help GPs diagnose headache, communicate MRI findings and better manage the remaining $97 \%$ of patients, who consult with headache but are not referred to specialists. Recent evidence from a study in Estonia showed changes in GP referral behaviour following an educational intervention. ${ }^{20}$ In our prior study, GPs reported improved confidence in managing headache in PC, following their participation in headache-specific training provided by a GP with Special Interest and training. ${ }^{13}$ Outcomes might be improved if neurologists or GPs with special interest in headache provide teaching for all local GPs, to enhance their confidence and competence when diagnosing and managing headache in PC. The added value in improved outcomes would extend not only for the $2 \%$ currently referred to headache specialists, but also to the remaining $97 \%$ managed without specialist input.

\section{Waiting for results}

We found that patients complained about long waits particularly to see specialists, which was avoided by PC direct access to imaging. However, we found clinicians, especially in GP clinics, did not appear to have clearly informed patients when and how they could access their results. Turnaround in reporting results depended on the neuroradiology department, and timeframes may not have been known by front-line clinicians in advance. Liaison between imaging and PC might be improved in the future. The lack of clarity about how to access their results disappointed expectations for some participants. As these investigations are important and expensive, having a protocol for communicating results and managing expected timelines, even when scans are normal, could improve this outcome.

\section{Patient anxiety}

Most patients had received their neuroradiology reports, and for over half it relieved their uncertainty. This is consistent with other studies. ${ }^{12} 1317$ Although for a minority, especially those with non-specific abnormalities, communicating these complex findings, without increasing patient anxiety, was difficult. As highlighted by past research, standardised reporting guidelines could help. ${ }^{8}$ In order to alleviate GP concerns about explaining complex reports to patients, more available neuroradiology advice could help to increase confidence in accuracy of diagnosis and provide specialist input in cases where needed. For example, the GPs in our previous study were not aware of a dedicated phone number to reach a neuroradiologist available to respond to queries. ${ }^{13}$ A Canadian study showed that poor GP-radiologist communication about the meaning of MRI reports can impact negatively on patient care. ${ }^{21}$ This is an ongoing challenge for neuroradiologists, neurologists and GPs.

\section{Usefulness of a normal scan}

Once a serious underlying problem had been eliminated by scanning, an equal number of patients in both neurology and GP pathways focused on the need to relieve pain. According to GPs, this is easier once an underlying concern for serious pathology such as a brain tumour is eliminated. ${ }^{13}$ Our evidence supports this, but without follow-up interviews this cannot be confirmed in the long run. Reviews of headache outcome and patients' experience suggest that this is a challenge in need of further research with a longer follow-up period. ${ }^{22} 23$ The management of continuing psychological comorbidity is probably the greatest challenge for patients and their clinicians. ${ }^{134}$ This suggests the need for more research on interventions that have proven successful in other areas like relaxation and cognitive behavioural therapy. ${ }^{25} 26$

The elephant in the room in most health services is how to deliver a service that improves outcomes, while reducing cost. Because of its prevalence and associated disability, headache incurs a high cost to individuals and, in a publicly funded service, to society. ${ }^{27}{ }^{28}$ Population neurologists have urged neurologists to demonstrate whether they actually can provide added value to outcomes when they provide care for particular conditions. ${ }^{29}$ It remains unclear whether outcomes are better with neurological referral for headache, and if these referrals are cost-effective. If more training was provided for GPs and those GPs with special 
interest in headache, patients might get added value at a reduced cost. ${ }^{30}$

\section{CONCLUSIONS}

This paper addresses the patients' view of one aspect of headache care, referral for imaging. We have previously found that the main reason for referral of people with headache was anxiety about serious pathology, with the patient and/or their GP wanting imaging. ${ }^{56}$ Howard $e t a l^{12}$ concluded that when patients or their GPs require reassurance from imaging, it is likely to be cost saving for specialists to arrange this. This is because when a specialist does not agree to request imaging, many patients asked their GP for referral to another specialist and obtain an MRI subsequently. GP direct access to imaging could reduce delay and avoid unnecessary referral to SC. This is supported by our study. However, delay in receiving imaging reports and communication of results could be improved.

The specific costs of this intervention are being evaluated separately through the Transforming Outcomes and Health Economics Through Imaging project. Whether GP open access will lead to a rising demand for investigation, and to what extent this forms part of a trend towards acknowledging patient choice, forms part of a continuing debate.

Acknowledgements Thanks for their input to Wendy Thomas, head of the Migraine Trust, and Carly Pearson. Above all, thanks to our participants who generously gave their time and thoughts on the research.

Contributors The study design was conceived by LR, with input from AM and RK, and from a migraine user group charity. RU led on data collection. AMc and RU led on analysis of data. All authors contributed to the interpretation. AMc wrote the paper with LR and additional comments from RU, GW, AM and RK. All authors have read and approved the final manuscript. LR is the chief investigator.

Funding This research was supported by a grant from the Guy's and St Thomas' Charity.

Disclaimer The views expressed in this manuscript are that of the authors and do not necessarily reflect the funders.

Competing interests None declared.

Patient consent for publication Not required.

Ethics approval The Health Research Authority granted ethical approval (REC ref: 16/NW/0326 North West - Liverpool Central Research Ethics Committee).

Provenance and peer review Not commissioned; externally peer reviewed.

Data sharing statement No data are available.

Open access This is an open access article distributed in accordance with the Creative Commons Attribution Non Commercial (CC BY-NC 4.0) license, which permits others to distribute, remix, adapt, build upon this work non-commercially, and license their derivative works on different terms, provided the original work is properly cited, appropriate credit is given, any changes made indicated, and the use is non-commercial. See: http://creativecommons.org/licenses/by-nc/4.0/.

\section{REFERENCES}

1. Latinovic R, Gulliford M, Ridsdale L. Headache and migraine in primary care: consultation, prescription, and referral rates in a large population. J Neurol Neurosurg Psychiatry 2006;77:385-7.

2. National Institute for Health and Clinical Excellence. Headaches in over 12s: diagnosis and management. 2012 https://www.nice.org.uk/ guidance/cg150/chapter/Recommendations\#assessment
3. Schon F, Hart P, Fernandez C. Is clinical neurology really so difficult? J Neurol Neurosurg Psychiatry 2002;72:557-9.

4. Ridsdale L, Mtandabari S, Noble A. 038 Referral patterns to neurology: past, present and future. J Neurol Neurosurg Psychiatry 2012;83:e1.203-e1.

5. Ridsdale L, Clark LV, Dowson AJ, et al. How do patients referred to neurologists for headache differ from those managed in primary care? Br J Gen Pract 2007;57:388-95.

6. Morgan M, Jenkins L, Ridsdale L. Patient pressure for referral for headache: a qualitative study of GPs' referral behaviour. $\mathrm{Br} J$ Gen Pract 2007;57:29-35.

7. Thomas R, Cook A, Main G, et al. Primary care access to computed tomography for chronic headache. Br J Gen Pract 2010;60:426-30.

8. Taylor TR, Evangelou N, Porter $\mathrm{H}$, et al. Primary care direct access MRI for the investigation of chronic headache. Clin Radio 2012;67:24-7.

9. Simpson GC, Forbes K, Teasdale E, et al. Impact of GP direct-access computerised tomography for the investigation of chronic daily headache. Br J Gen Pract 2010;60:897-901.

10. Morris Z, Whiteley WN, Longstreth WT, et al. Incidental findings on brain magnetic resonance imaging: systematic review and metaanalysis. BMJ 2009;339:b3016.

11. Hayward R. VOMIT (victims of modern imaging technology) - an acronym for our times. BMJ 2003;326:1273.

12. Howard L, Wessely S, Leese M, et al. Are investigations anxiolytic or anxiogenic? A randomised controlled trial of neuroimaging to provide reassurance in chronic daily headache. $J$ Neurol Neurosurg Psychiatry 2005;76:1558-64.

13. Underwood R, Kilner R, Ridsdale L. Primary care management of headaches and how direct-access MRI fits: a qualitative study of UK general practitioners' views. BMJ Open 2017;7:e018169.

14. Braun V, Clarke V. Using thematic analysis in psychology. Qual Res Psychol 2006;3:77-101.

15. Guest G, Bunce A, Johnson L. How many interviews are enough?: an experiment with data saturation and variability. Field Methods 2006;18:59-82.

16. Tong A, Sainsbury P, Craig J. Consolidated criteria for reporting qualitative research (COREQ): a 32-item checklist for interviews and focus groups. Int J Qual Health Care 2007;19:349-57.

17. Ridsdale L, J. Noble A, Nadeem F, et al. Headache: the patient's view. J Neurol Neurosurg 2014;01:5.

18. Apthorp LA, Daly CA, Morrison ID, et al. Direct access MRI for general practitioners - influence on patient management. Clin Radiol 1998;53:58-60.

19. White PM, Halliday-Pegg JC, Collie DA. Open access neuroimaging for general practitioners - diagnostic yield and influence on patient management. Br J Gen Pract 2002;52:33-5.

20. Braschinsky M, Haldre S, Kals M, et al. Structured education can improve primary-care management of headache: the first empirical evidence, from a controlled interventional study. J Headache Pain 2016;17:24.

21. You JJ, Levinson W, Laupacis A. Attitudes of family physicians, specialists and radiologists about the use of computed tomography and magnetic resonance imaging in Ontario. Healthc Policy 2009;5:54-65.

22. Nichols VP, Ellard DR, Griffiths FE, et al. The lived experience of chronic headache: a systematic review and synthesis of the qualitative literature. BMJ Open 2017;7:e019929.

23. Probyn $\mathrm{K}$, Bowers $\mathrm{H}$, Caldwell $\mathrm{F}$, et al. Prognostic factors for chronic headache: a systematic review. Neurology 2017;89:291-301.

24. Stone J, Evans RW. Functional/psychogenic neurological symptoms and headache. Headache 2011;51:781-8.

25. Sullivan A, Cousins S, Ridsdale L. Psychological interventions for migraine: a systematic review. J Neurol 2016;263:2369-77.

26. Probyn K, Bowers H, Mistry D, et al. Non-pharmacological selfmanagement for people living with migraine or tension-type headache: a systematic review including analysis of intervention components. BMJ Open 2017;7:e016670.

27. McCrone P, Seed PT, Dowson AJ, et al. Service use and costs for people with headache: a UK primary care study. $J$ Headache Pain 2011;12:617-23.

28. Osumili B, McCrone P, Cousins S, et al. The economic cost of patients with migraine headache referred to specialist clinics. Headache 2018;58:287-94.

29. Morrish $P$. The changing relationship between neurology and general practice in the UK. Br J Gen Pract 2009;59:599-604.

30. Ridsdale L, Doherty J, McCrone P, et al. A new GP with special interest headache service: observational study. $\mathrm{Br} J$ Gen Pract 2008;58:478-83. 